9 Joist JH. Hemostatic abnormalities in liver disease. In: Colman RW, Hirsh J, Marder VJ, Salzman EW, eds. Hemostasis and thrombosis: basic principles and clinical practice. 2nd ed. Philadelphia: Lippincott, 1987:861-72.

10 Stein SF, Harker LA. Kinetic and functional studies of platelets, fibrinogen, and plasminogen in patients with hepatic cirrhosis. I L.ab Clin Med 1982;99:217-30.

11 Ballard HS, Marcus AJ. Platelet aggregation in portal cirrhosis. Arch Intern Med 1976;136:316-9.

12 Hallen A, Nilsson IM. Coagulation studies in liver disease. Thrombosis $e$ Diathesis Haemorrhagica 1964;11:51-63.

13 Burroughs AK, Scheuer PJ. Liver biopsy-indications and procedure. Medicine International 1986;2:1174-6.

14 Picinino F, Sagnelli E, Pasquale G, Giusty G. Complications following percutaneous liver biopsv. A multicentre retrospective study on 68,276 biopsies. I Hepatol 1986;2:165-73.

15 Wildhirt E, Moller E. Erfhrungen bei nahezu 20,000 leberblindpunktionen. Med Klin 1981;76:254-6.

16 Ewe K. Bleeding after liver biopsy does not correlate with indices of peripheral coagulation. Dig Dis Si 1981;26:388-93.

17 Mielke $\mathrm{CH}$. Measurement of the bleeding time. International committe communications. Thromb Haemost 1984;52:210-1.

18 Rubin $\mathrm{MH}$, Weston MJ, Langley PJ, White Y, Williams R. Platelet function in chronic liver disease: relationship to disease severity. Dig Dis Sc 1979;24:197-202

19 De Noronha R, Taylor B, Greaves $M$, Triger DR. Thrombocytopenia in liver disease: an immune phenomenon? [Abstract]. Gut 1990;30:A1495.
20 Owen JS, Hutton RA, Dav RC, Bruckdorfer R, McIntyre N. Platelet lipid composition and platelet aggregation in human liver disease. 7 Lipid Res 1981:22:423-30.

21 Desai K, Bagget C, Bellamy MF, Mistry P, Burroughs AK, Owen JS. Inhibition of platelet aggregation by abnormal high density lipoprotein particles in plasma from patients with hepatic cirrhosis. Lancet 1989;i:693-5.

22 Minuk GY. Sutherland LR, Wiseman DA, McDonald FR, Ding DL. Prospective study of the incidence of ultra-sound detected intrahepatic and subcapular hematomas in patients randomised to 6 or 24 hours of bed rest after percutaneous liver biopsy. Gastroenterologv 1987;92:290-3.

23 Mannucci PM, Franchi F, Dioguardi N. Correction of abnormal coagulation in chronic liver disease by combined use of fresh frozen plasma and prothrombin complex concentrations. Lancet 1976;ii:542-5.

24 Burroughs AK, Mathews K, Qadiri M, et al. Desmopressin and bleeding time in patients with cirrhosis. Br Med f 1985;291:1377-81.

25 Mannucci PM, Vincente $V$, Vianello $L$, at al Controlled trial of desmopressin in liver cirrhosis and other conditions associated with a prolonged bleding in liver cirrhosis and other cond

26 Burroughs AK, Taylor L, Sprengers D, Hutton RA, McIntyre N, Kernoff P Haemostatic changes in cirrhosis after repeated doses of desamino D arginine vasopressin [Abstract]. Thromb Haemost 1987;58:365.

27 Sprengers D, Grech P, McCormick PA, McIntyte N, Burroughs AK. Is bleeding time a useful test in cirrhotic patients? [Abstract]. Gut 1987;28: A 1348 .

(Accepted 20 April 1990)

\title{
Livèr transplantation in patients with alcoholic cirrhosis: selection criteria and rates of survival and relapse
}

\author{
George L A Bird, John G O'Grady, Felicity A H Harvey, Roy Y Calne, Roger Williams
}

\begin{abstract}
Objective-To evaluate the outcome of liver transplantation in patients with alcoholic cirrhosis with respect to selection criteria, survival, and evidence suggesting a return to harmful drinking.

Design-Nine year retrospective study.

Setting-Cambridge and King's College Hospital liver transplant programme.
\end{abstract}

Subjects - 24 Patients (three women, 21 men) with alcoholic cirrhosis.

Main outcome measures-Survival, rehabilitation, and clinical and laboratory evidence of a return to harmful drinking after transplantation.

Results - 15 Patients were selected for transplantation because of repeated admission to hospital for the complications of advanced portal hypertension despite abstinence, and six because they had a hepatocellular carcinoma superimposed on alcoholic cirrhosis. Three patients who were not abstinent received transplants. The one year survival rate was $66 \%$, and of the 18 patients surviving at least three months, 17 had been rehabilitated. In three patients laboratory variables and histological examination of the liver suggested a return to drinking, though they did not admit to taking alcohol. These patients represented the only cases in the series that were not abstinent before transplantation.

Conclusions-The survival and rehabilitation of patients who received transplants for alcoholic cirrhosis compared favourably with those of patients who received transplants for cirrhosis of other aetiology. The criteria for selection for liver transplantation in patients with alcoholic cirrhosis should include recurrent complications related to severe portal hypertension despite maximum medical treatment in addition to a minimum period of six months of abstinence before transplantation.

\section{Introduction}

Alcoholic cirrhosis has been accepted as an indication for liver transplantation since the National Institutes of Health consensus development panel stated that "It may be considered for patients in whom evidence of progressive liver failure develops despite medical treatment and abstinence from alcohol." Many potential transplant recipients, however, are disqualified by the coexistence of neurological and cardiovascular disease induced by alcohol, together with a poor nutritional state. The possibility that there may be a return to alcohol misuse after transplantation has also given rise to negative attitudes to liver transplantation in this group of patients.

A study in Pittsburgh recently found that the outcome of transplantation among patients with alcoholic cirrhosis was as good as that among patients with other types of liver disease, even though abstinence was not a requirement in selecting patients. ${ }^{3}$ Such a relaxation of the criteria for selection could lead to a considerable increase in the number of candidates for transplantation and raises questions concerning priorities in allocating donated organs. ${ }^{2}$ We reviewed the outcome among patients with alcoholic liver disease who had received a liver transplant at Addenbrooke's or King's College Hospitals during the past nine years.

\section{Patients and methods}

Twenty four patients with alcoholic liver disease (three women, 21 men; age range 29-59, median 48) received liver transplants between 1 January 1980 and 1 December 1989. Patients were generally considered for transplantation if, despite abstinence, the presence of severe portal hypertension suggested a poor prognosis. ${ }^{4}$ Contraindications included extrahepatic disease induced by alcohol (cerebral dysfunction other than encephalopathy, cardiomyopathy, pancreatitis) or severe malnutrition. Survival was analysed by the Kaplan-Meier method.

\section{Results}

\section{CRITERIA FOR SELECTION}

The indication for transplantation in six patients was hepatocellular carcinoma superimposed on alcoholic cirrhosis. The other 18 patients had been admitted to hospital several times for management of ascites, hepatic encephalopathy, and recurrent variceal bleeding. Major impairment of hepatocellular function, as judged by laboratory tests was also present (table I) 
All patients had a long history of heavy alcohol consumption, but the duration of clinically documented liver disease was wide ranging (table II). Among the patients who had stopped drinking, the duration of abstinence ranged from three months to 12 years (median two years). In the three patients who were not abstinent the decision to proceed to transplantation was made on the basis of the rapid progression of liver disease (over one to four years), the youth of the patients, and the commitment of each patient to a long term programme of treatment for alcohol dependence.

TABLE I-Clinical and laboratory characteristics of 24 patients with alcoholic liver disease before liver transplantation

\begin{tabular}{lc}
\hline Characteristics & No \\
\hline No of patients with: & 19 \\
$\quad$ Ascites & 9 \\
Previous episodes of hepatic encephalopathy & 7 \\
Recurrent variceal bleeding & \\
Abdominal pain (secondary to hepatocellular & 2 \\
$\quad$ carcinoma) & $5(0-20)$ \\
Median (range): & $40(6-152)$ \\
Prothrombin time (seconds prolonged) & $30(20-38)$ \\
Serum bilirubin $(\mu \mathrm{mol} / \mathrm{l})$ & \\
\hline
\end{tabular}

TABLE II-Duration of alcohol consumption and documented liver disease in patients undergoing liver transplantation for alcoholic cirrhosis

\begin{tabular}{lc}
\hline & Median (range) \\
\hline Duration of alcohol consumption & 18 years $(5-30$ years $)$ \\
Daily intake of alcohol & $300 \mathrm{~g}(80-600 \mathrm{~g})$ \\
Duration of documented liver disease & 3 years $(6$ months -19 \\
& years $)$ \\
\hline
\end{tabular}

RATE OF SURVIVAL

The overall one year survival rate (follow up from four months to seven years) was $66 \%$, compared with a rate of $56 \%$ among 324 patients with other types of cirrhosis who had received transplants since 1980. Six patients died within five weeks after liver transplantation, and of the 18 patients surviving at least three months, three died subsequently from chronic rejection, squamous cell carcinoma of the neck, and recurrence of hepatocellular carcinoma.

In all patients histological examination of the explanted liver confirmed the presence of alcoholic cirrhosis, and the livers of three patients, two of whom were abstinent, showed features of acute alcoholic hepatitis. Of the three patients with alcoholic hepatitis, two were alive 18 and 30 months after transplantation.

Of the six patients with hepatocellular carcinoma and alcoholic cirrhosis, two died within five weeks after the transplantation and one died from recurrence of the tumour four years after receiving the transplant. The remaining three patients survived with no evidence of tumour recurrence.

\section{REHABILITATION AND RELAPSE}

All but one of the 18 patients who survived at least three months were considered to have resumed a satisfactory degree of social and occupational function, with a return to full domestic duties or employment. In the patient in whom rehabilitation was not achieved and three others, a raised erythrocyte mean cell volume was present within one year after the transplantation despite good graft function and nutritional state. Three of these patients denied drinking and also gave negative responses to a screening questionnaire for alcohol misuse, although one of them had a poor record of attendance and bruises from recurrent falls. Another patient developed a florid complexion and a tremor, although her requirement for prednisolone and cyclosporin was low and other possible cushingoid side effects were absent. Histological examination of the liver of this patient two years after transplantation showed alcoholic hyaline, fatty change, and extensive fibrosis highly suggestive of damage caused by alcohol.

Determination of the ratio of the activities of serum $\gamma$-glutamyltransferase to serum alkaline phosphatase showed that at 12 months after transplantation three of the four patients with raised mean cell volumes had ratios $>1 \cdot 8$, considerably higher than those patients who had normal mean cell volumes, whether they had received a transplant for alcoholic cirrhosis or other liver disease. No correlation was found between the mean cell volume and serum $\gamma$-glutamyltransferase activity in individual patients who had received a transplant for alcoholic or other liver diseases. The three patients in whom there was evidence of a return to alcohol consumption were the only three in the series who were not abstinent before transplantation.

\section{Discussion}

We have shown that the rates of survival and rehabilitation of patients undergoing liver transplantation for alcoholic cirrhosis were as good as in patients with cirrhosis of other aetiologies, which agrees with the study from Pittsburgh. ${ }^{3}$ Although recurrence of tumours is now recognised to be common after transplantation for hepatocellular carcinoma, this occurred in only one case. The inclusion of the six patients with hepatocellular carcinoma in our analysis was therefore not an important adverse influence on survival.

The 18 patients selected for transplantation who did not have hepatocellular carcinoma represented less than $5 \%$ of the total number of patients who received transplants during the period studied, and this reflects the fairly small proportion of patients with alcoholic liver disease eligible for transplantation. All 18 patients had been admitted to hospital several times for complications arising from severe portal hypertension and had had clinical and laboratory features indicative of a poor prognosis. ${ }^{+}$Interestingly, histological evidence of alcoholic hepatitis was present in the hepatectomy specimens of three patients, two of whom had allegedly been abstinent before transplantation. A longer period of abstinence in these two patients may have resulted in improvement due to spontaneous resolution of the hepatitis.

Laboratory and clinical evidence of a return to heavy drinking was found in four patients, although only one of them admitted to drinking. Mild abnormalities in the results of tests of liver function are common after transplantation and biochemical abnormalities indicative of heavy alcohol consumption in patients without liver transplants are more difficult to interpret in liver transplant recipients. Although there was no correlation between suspected drinking and absolute activities of $\gamma$-glutamyltransferase after transplantation, the presence of a ratio of $\gamma$-glutamyltransferase to alkaline phosphatase greater than 1.8 one year after transplantation in three of the four patients suspected of drinking because of clinical findings and a raised mean cell volume suggested that this ratio could be a useful indicator of high alcohol intake after liver transplantation.

The finding that three of the four patients in whom evidence was found for a return to alcohol were not abstinent at the time of the transplant operation contrasts with the Pittsburgh series, in which abstinence was not required yet no patient returned to excessive drinking. Another study that did not require patients to be abstinent before transplantation did not report the number of patients who relapsed after the operation.

Our results suggest that liver transplantation should not be considered as a treatment for alcohol dependence 
in itself, and the criteria for selecting patients under normal circumstances should include a period of abstinence of at least six months in addition to an assessment of alcohol dependence. With the availability of organs likely to become an increasingly limiting factor as the number of liver transplant operations performed increases, such a policy would also be more likely to be acceptable to the general public and to the families granting permission for organ donation.
Anonymous. Liver transplantation. FAMA 1983;250:2961-4.

Neuberger JM. Transplantation for alcoholic liver disease. Br Med $f 1989$ 299:693.

3 Kumar S, Stauber RE, Gavaler JS, et al. Orthotopic liver transplantation for alcoholic liver disease. Hepatologv 1990;11:159-64

4 Christensen E, Schlichting P, Kragh-Andersen P, et al. Updating prognosis and therapeutic effect evaluation in cirrhosis with Cox's multiple regressio model for time-dependent variables. Scand $\mathcal{F}$ Gastroenterol 1986;21:163-74 5 Mayfield D, MacLeod G, Hall P. The CAGE questionnaire: validation of a new alcoholism screening instrument. Am f Psychiatry 1974;131:1121-3.

6 Lucey MR, Merion RM, Henley KS, et al. Selection of patients with alcoholic liver disease for orthotopic liver transplantation. Hepatology 1989;10:572.

(Accepted 9 May 1990)

\title{
Hospital care for the elderly in the final year of life: a population based study
}

\author{
J Henderson, M J Goldacre, $M$ Griffith
}

\begin{abstract}
Objectives-To determine whether among people aged 65 and over those who died at advanced old age spent more of their last year of life in hospital than those who died younger, and whether the increase in longevity in the elderly between 1976 and 1985 was accompanied by increased time spent in hospital in the last year of life.
\end{abstract}

Design-Linkage of death records to abstracts of records of hospital inpatient care in the preceding year of patients' lives.

Setting-Six health districts in England covered by the Oxford record linkage study.

Results-People who died at advanced ages (85 and over) were less likely than people who died at younger ages $(65-84)$ to have been admitted to hospital in the last year of life. Once admitted the very old tended to spend longer in hospital than others. The mean total time spent in hospital by the elderly in the year before death (based on all deaths including those among people not admitted at all) showed no appreciable change over time. The median time in hospital based on all deaths increased by about three days between 1976 and 1985 . During that time there was a gain in life expectancy in the population of about one year from the age of 65 .

Conclusion-The gain in life expectancy in this population was not at the expense of any substantial increase in time spent in hospital in the final year of life.

\section{Introduction}

Life expectancy after the age of 65 continues to increase. In England and Wales between 1975 and 1985 it increased from $12 \cdot 4$ to $13 \cdot 4$ years for men and from $16 \cdot 4$ to $17 \cdot 5$ years for women. ${ }^{1}$ It has been suggested that such extra years of life gained by the elderly may represent increased duration of disability and dependency rather than longer active life..$^{2.5}$ This has been termed the "failures of success." Fries suggested that as longevity increases there may also be postponement of the onset of disability. ${ }^{6}$ This possibility of "compression of morbidity" carries the implication that with increases in longevity the need for medical care for the elderly at a given age may actually decrease.

This study was undertaken to estimate the extent to which elderly people spent time in hospital in the year before death. Its main purpose was to determine the extent to which increases in life span were accompanied by increased time in hospital in the final year of life. Time spent in hospital by the elderly is influenced not only by levels of ill health but also by such factors as family and social support, medical policies in respect of admission and duration of stay, the availability of hospital facilities, and the availability of alternative forms of residential care. Whatever the reasons for hospital care in any particular case we have assumed that in general elderly people prefer to be well enough and supported enough such that they do not spend long periods in hospital.

\section{Methods}

Data from the Oxford record linkage study were used. This is a series of brief abstracts of hospital records and death records collected such that it is possible to link together successive records relating to the same person. Data collection for the Oxford record linkage study began in 1963 in part of Oxfordshire, and coverage expanded over the years to include six districts of the Oxford region from 1975 (population 1.9 million).

Our analysis included records of all residents of the six districts who died over the age of 64 between 1976 and 1985 . Their death records were identified and the general hospital files searched for all episodes of hospital care experienced by these people in the 365 days before death. All episodes of inpatient care in general hospitals were included; data on psychiatric care were not included. Data on residential and nursing homes were not available. The lengths of stay in all episodes of hospital care were linked together and summed to give total days in hospital in the final year of life.

The percentage of the study population who had been admitted to hospital at least once in the year before death was calculated. Total days in hospital were calculated, firstly, as means and medians based on all people who were admitted to hospital in the last year of life and, secondly, as means and medians based on the total population aged over 64 who were within a year of death and who were resident within the area covered by the study. Mean and median values were calculated because of the highly skewed distributions of times spent in hospital. The analyses were repeated excluding hospital admissions in the last week of life to identify the separate influence of such terminal care. Statistical significance of the trends over time was calculated by $\chi^{2}$ test for trend in analysing admission rates and linear regression for analysis of trends in mean and median lengths of stay.

\section{Results}

ADMISSIONS IN YEAR BEFORE DEATH

Death records were identified for 141817 people aged 65 and over. The proportion of elderly people 